\title{
The role of the pion cloud in electroproduction of the $\Delta(1232)$
}

\author{
M. Fiolhais 时, B. Golli b. \\ a Department of Physics, University of Coimbra, 3000 Coimbra, Portugal \\ b Faculty of Education, University of Ljubljana, Ljubljana, Slovenia \\ c J. Stefan Institute, Jamova 39, 61111 Ljubljana, Slovenia
}

\begin{abstract}
We calculate the ratios $E 2 / M 1$ and $C 2 / M 1$ of the multipole amplitudes for electroproduction of the $\Delta(1232)$ in the range of photon virtuality $0<-K^{2}<1 \mathrm{GeV}^{2}$ in a chiral chromodielectric model and a linear $\sigma$ model. We find that relatively large experimental values can be explained in terms of the pion contribution alone; the contribution arising from dstate quark admixture remains below $10 \%$. We describe the pion cloud as a coherent state and use spin and isospin projection to obtain the physical nucleon and the $\Delta$. The $A_{1 / 2}$ and $A_{3 / 2}$ amplitudes are reasonably well reproduced in the $\sigma$-model; in the chromodielectric model, however, they are a factor of two too small.
\end{abstract}

(PACS 12.35H, 13.60P)

The new (e, $\left.\mathrm{e}^{\prime} \pi\right)$ experiments in Mainz and at MIT/Bates, and those planned at CEBAF have considerably raised the interest in theoretical calculations of the amplitudes for electroproduction of low lying baryon resonances. Of particular interest are indications for relatively large quadrupole $E 2$ (or $E_{1+}$ ) and $C 2$ $\left(S_{1+}\right)$ amplitudes in the vicinity of the $\Delta(1232)$ resonance which mix with the leading $M 1\left(M_{1+}\right)$ amplitude. The quark model calculations, assuming d-state quark admixtures in the nucleon and the $\Delta$, generally lead to much too small values [1]. On the other hand, large values can be reproduced by assuming a sufficiently strong p-wave pion field surrounding three valence quarks. In the Cloudy Bag Model (CBM), a reasonably good agreement with the measured amplitudes for photoproduction has been obtained with $R=0.6-0.8 \mathrm{fm}$ [4, 5]. However, at these bag radii the pion field is already so strong that the use of perturbative approach is questionable. It is therefore interesting to study the

1 E-mail: tmanuel@hydra.ci.uc.pt

2 E-mail: bojan.golli@ijs.si

3 E-mail: simon.sirca@ijs.si

Preprint submitted to Elsevier Preprint

29 January 1996 
process in the framework of a nonperturbative approach such as using coherent states to describe the pion cloud.

Our aim here is to compute the behaviour of the quadrupole amplitudes at low photon virtualities $K^{2}$ (where the concept of the pion as an elementary excitation is still sensible), and investigate which features do and which do not depend on details of a particular model. We therefore study the process in the chiral chromodielectric model (CDM) and the linear $\sigma$ model (LSM), which, though providing different pictures for the nucleon, both account for a good description of its static properties [6]. In these models the nucleon and the $\Delta$ are described as chiral solitons resulting from the non-linear interactions between quarks and scalar-isoscalar $(\sigma)$ and pseudoscalar-isovector $(\vec{\pi})$ mesons. The CDM contains, in addition, a scalar-isoscalar chiral singlet field $\chi$ which, through the peculiar way it couples to the quarks, provides a mechanism for confinement. In the LSM the pion field in the nucleon is relatively strong as a consequence of the topologically nontrivial solution for the meson fields resembling in many aspects the Skyrmion solution, while in the CDM it is weaker and similar to the solution in the CBM (for $R$ above $1 \mathrm{fm}$ ).

The Lagrangian of the models can be written as [6]

$$
\mathcal{L}=\mathcal{L}_{q}+\mathcal{L}_{\sigma, \pi}+\mathcal{L}_{q-\text { meson }}+\mathcal{L}_{\chi}
$$

where

$$
\mathcal{L}_{q}=\mathrm{i} \bar{\psi} \gamma^{\mu} \partial_{\mu} \psi, \quad \mathcal{L}_{\sigma, \pi}=\frac{1}{2} \partial_{\mu} \hat{\sigma} \partial^{\mu} \hat{\sigma}+\frac{1}{2} \partial_{\mu} \hat{\vec{\pi}} \cdot \partial^{\mu} \hat{\vec{\pi}}-\mathcal{U}\left(\hat{\vec{\pi}}^{2}+\hat{\sigma}^{2}\right)
$$

$\mathcal{U}\left(\hat{\vec{\pi}}^{2}+\hat{\sigma}^{2}\right)$ being the usual Mexican hat potential, and the quark meson interaction is given by

$$
\mathcal{L}_{q-\text { meson }}=\frac{g}{\chi^{p}} \bar{\psi}\left(\hat{\sigma}+\mathrm{i} \vec{\tau} \cdot \hat{\vec{\pi}} \gamma_{5}\right) \psi
$$

In the LSM, $p=0$; in the CDM we take $p=1$. The last term in (1) - absent in the LSM model - contains the kinetic and the potential piece for the $\chi$-field:

$$
\mathcal{L}_{\chi}=\frac{1}{2} \partial_{\mu} \hat{\chi} \partial^{\mu} \hat{\chi}-\frac{1}{2} M_{\chi}^{2} \hat{\chi}^{2}
$$

The second term on the RHS is just the mass term for the $\chi$ field. Other versions of the CDM consider a quartic potential as well as other powers $p$ in (3). By taking just the mass term and $p=1$ the confinement is imposed in the smoothest way, which seems to be the most appropriate choice for the quark matter sector of the CDM [7]. 
Using coherent states to describe the pion and the $\sigma$-meson clouds with the "hedgehog" ansatz [8] for the pion field and the three valence quarks, the intrinsic state takes the form [9]

$$
\begin{aligned}
|H\rangle= & \mathcal{N} \exp \left\{\sum_{m}(-1)^{1-m} \int \mathrm{d} k \sqrt{2 \pi \omega_{k} / 3} k \pi(k) a_{1 m,-m}^{\dagger}(k)\right\} \\
& \times \exp \left\{\int \mathrm{d} k \sqrt{2 \pi \widetilde{\omega}_{k}} k \sigma(k) \widetilde{a}^{\dagger}(k)\right\}\left(b_{u \downarrow}^{\dagger}-b_{d \uparrow}^{\dagger}\right)^{3}|0\rangle .
\end{aligned}
$$

Here $\widetilde{a}^{\dagger}(k)$ and $a_{l m t}^{\dagger}(k)$ are the creation operators for the $\sigma$-meson and the pion, respectively, in the spherical basis and $t$ is the third component of isospin, $\omega_{k}^{2}=k^{2}+m_{\pi}^{2}$ and $\widetilde{\omega}_{k}^{2}=k^{2}+m_{\sigma}^{2}, b_{u \downarrow}^{\dagger}\left(b_{d \uparrow}^{\dagger}\right)$ is the creation operator for the 'up' ('down') quark in the 1 s-state with the third component of spin $-\frac{1}{2}\left(\frac{1}{2}\right)$. Only swave $\sigma$-mesons and p-wave pions are coupled to the quark core. The functions $\sigma(k)$ and $\pi(k)$ are related to the expectation values of the field operators as $\langle H|\hat{\sigma}(\boldsymbol{r})| H\rangle=\sigma(r)$ and $\left\langle H\left|\hat{\pi}_{t}(\boldsymbol{r})\right| H\right\rangle=\pi(r) \hat{r}_{-t}$, where the profiles $\sigma(r)$ and $\pi(r)$ are the Fourier transforms of $\sigma(k)$ and $\pi(k)$, respectively. The physical states are obtained by performing the Peierls-Yoccoz projection

$$
\left|J=T ; M_{T}, M_{J}\right\rangle=\mathcal{N}^{\prime}(-1)^{J+M_{T}} P_{M_{J},-M_{T}}^{J}|H\rangle .
$$

In the CDM the $\chi$ field is included in the same way as the $\sigma$ field and is not affected by projection. The meson and quark profiles are determined selfconsistently using variation after projection.

The free parameters of the models have been chosen by requiring that the calculated static properties of the nucleon agree best with the experimental values. In the LSM model we use $g=5.0$. In the considered version of the CDM the results are predominantly sensitive to the quantity $G=\sqrt{g M_{\chi}}$; we take $G=0.2 \mathrm{GeV}$ (and $g=0.03 \mathrm{GeV}$ ). We have checked that our results depend very weakly on the variations of these parameters. The models contain three other parameters, the chiral meson masses and the pion decay constant, which are fixed to the following values: $m_{\pi}=0.14 \mathrm{GeV}, m_{\sigma}=1.2 \mathrm{GeV}$, $f_{\pi}=0.093 \mathrm{GeV}$.

It is known that in these models the $\Delta-\mathrm{N}$ mass splitting is too small (typically $160 \mathrm{MeV}$ in the LSM and only $60 \mathrm{MeV}$ in the CDM). It has been suggested [10] that this deficiency can be cured through the ' $t$ Hooft interaction which is attractive for the quarks in the bare nucleon and absent for the bare $\Delta$. In our calculation we include this effect as well as the effect of residual chromomagnetic interaction by considering different masses for the bare nucleon and $\Delta$ in order to reproduce the physical $\Delta-\mathrm{N}$ splitting. The relevant parameter is the difference between the bare masses, $\varepsilon_{\Delta \mathrm{N}}$. While it has only little effect on the pion clouds in the LSM, in the CDM it considerably increases the strength 
of the pion field in the $\Delta$, whereas that in the nucleon is slightly decreased. This can be easily understood: in the variational calculation of the physical $\Delta$ the energetically less favourable bare $\Delta$ (otherwise the dominant contribution) is being suppressed in favour of the configuration with one (or more) pions around the bare nucleon.

The helicity amplitudes $A_{1 / 2}$ and $A_{3 / 2}$ for electroproduction of the $\Delta$ resonance are defined as

$$
A_{\lambda}=-\frac{e}{\sqrt{2 k_{W}}}\left\langle\Delta ; \frac{1}{2}, \lambda\left|\int \mathrm{d}^{3} \boldsymbol{r} \boldsymbol{\epsilon} \cdot \widehat{\boldsymbol{J}}(\boldsymbol{r}) \mathrm{e}^{\mathrm{i} \boldsymbol{k} \boldsymbol{r}}\right| \mathrm{N} ; \frac{1}{2}, \lambda-1\right\rangle,
$$

where $e=\sqrt{4 \pi \alpha}, \boldsymbol{\epsilon}=-1 / \sqrt{2}(1, \mathrm{i}, 0)$ and the photon three-momentum $\boldsymbol{k}$ is given by conservation of energy as

$$
|\boldsymbol{k}|^{2}=\omega^{2}-K^{2} \equiv k^{2}=\left[\frac{M_{\Delta}^{2}+M_{\mathrm{N}}^{2}-K^{2}}{2 M_{\Delta}}\right]^{2}-M_{\mathrm{N}}^{2} .
$$

We adopt here the convention [11] that for virtual photons the factor $1 / \sqrt{2 \omega}$ in the expansion of the photon field is replaced by $1 / \sqrt{2 k_{W}}, k_{W}$ being the value of $k$ at the photon point $\left(K^{2}=0\right)$. $\square$ In practical calculation it is convenient to expand the operator in (7) in terms of electric and magnetic multipoles; the relevant quantities are

$$
\begin{gathered}
M^{\mathrm{M} 1}=-\frac{3}{2} \int \mathrm{d}^{3} \boldsymbol{r}\left\langle\Delta ; \frac{1}{2}, \frac{1}{2}\left|(\hat{\boldsymbol{r}} \times \widehat{\boldsymbol{J}})_{1}\right| \mathrm{N} ; \frac{1}{2},-\frac{1}{2}\right\rangle j_{1}(k r), \\
M^{\mathrm{E} 2}=-\frac{\sqrt{10 \pi}}{k} \int \mathrm{d}^{3} \boldsymbol{r}\left\langle\Delta ; \frac{1}{2}, \frac{1}{2}\left|\left[\boldsymbol{\nabla} \times j_{2}(k r) \boldsymbol{Y}_{22}^{1}(\hat{\boldsymbol{r}})\right] \cdot \widehat{\boldsymbol{J}}(\boldsymbol{r})\right| \mathrm{N} ; \frac{1}{2},-\frac{1}{2}\right\rangle .
\end{gathered}
$$

The E2 multipole (10) can be expressed in terms of the charge operator using current conservation as

$$
\begin{aligned}
M^{\mathrm{E} 2}= & \frac{\sqrt{15 \pi}}{3} \int \mathrm{d}^{3} \boldsymbol{r}\left\langle\Delta ; \frac{1}{2}, \frac{1}{2}\right|\left[\frac{\omega}{k} \widehat{\rho}(\boldsymbol{r}) \frac{\partial}{\partial r} r j_{2}(k r)\right. \\
& \left.-\mathrm{i} k \boldsymbol{r} \cdot \widehat{\boldsymbol{J}}(\boldsymbol{r}) j_{2}(k r)\right]\left|\mathrm{N} ; \frac{1}{2},-\frac{1}{2}\right\rangle Y_{21}(\hat{\boldsymbol{r}}) .
\end{aligned}
$$

Defining the Coulomb quadrupole

$$
M^{\mathrm{C} 2}=-\sqrt{20 \pi} \int \mathrm{d}^{3} \boldsymbol{r}\left\langle\Delta ; \frac{1}{2}, \frac{1}{2}|\widehat{\rho}(\boldsymbol{r})| \mathrm{N} ; \frac{1}{2}, \frac{1}{2}\right\rangle Y_{20}(\hat{\boldsymbol{r}}) j_{2}(k r),
$$

1 Had we kept the factor $1 / \sqrt{2 \omega}$ in (7), the amplitude would have diverged for $K^{2}=M_{\mathrm{N}}^{2}-M_{\Delta}^{2}$. Since the amplitude for electroproduction of the $\Delta$ is not directly observable, one is allowed to introduce such a convention (together with a corresponding convention for the decay amplitude) in order to avoid this divergence. 
the $E 2 / M 1$ and $C 2 / M 1$ ratios are given as

$$
\begin{aligned}
& \frac{E 2}{M 1}=\frac{1}{3} \frac{M^{\mathrm{E} 2}}{M^{\mathrm{M} 1}}, \\
& \frac{C 2}{M 1}=\frac{1}{2 \sqrt{2}} \frac{M^{\mathrm{C} 2}}{M^{\mathrm{M} 1}} .
\end{aligned}
$$

Note that in the limit $k \rightarrow 0$, ratios (13) and (14) are equal. The current and the charge density operators contain the quark and the pion part:

$$
\begin{aligned}
\widehat{\boldsymbol{J}} & =\bar{\psi} \gamma\left(\frac{1}{6}+\frac{1}{2} \tau_{3}\right) \psi-(\hat{\vec{\pi}} \times \boldsymbol{\nabla} \hat{\vec{\pi}})_{3} \\
\widehat{\rho}(\boldsymbol{r}) & =\bar{\psi} \gamma_{0}\left(\frac{1}{6}+\frac{1}{2} \tau_{3}\right) \psi+(\hat{\vec{\pi}} \times \hat{\overrightarrow{\vec{P}}})_{3},
\end{aligned}
$$

where $\hat{\vec{P}}_{\pi}$ stands for the canonically conjugate field. Using the grand spin symmetry of the hedgehog, the evaluation of transition matrix elements between the projected states representing the nucleon and the $\Delta$ is considerably simplified; details of the calculation technique as well as a discussion of the validity of the hedgehog approximation can be found in [12].

If we assume, as in (6), that the quarks occupy only the lowest s-state, there is no quark contribution to the $E 2$ and $C 2$ amplitudes. However, the interaction (3) between the p-wave pions and the quarks mixes the s-state and the d-state $(j=3 / 2)$ quarks. It is then possible that the $E 2$ and $C 2$ photons couple directly to quarks yielding nonvanishing contributions to the $E 2$ and $C 2$ amplitudes. Such a contribution can be calculated in a straightforward way in the CDM; in the LSM this is directly not possible since there are no bound d-states in this model. We calculated this effect in the CDM by solving the Dirac equation in the background $\chi$ and meson fields. Since the interaction responsible for the mixing is weak, it is enough to take into account only the lowest excited state and calculate the amplitudes in the first order perturbation theory. In contrast to the pion contribution, the quark part is much more sensitive to the detailed structure of the states, in particular to the bare $\Delta-\mathrm{N}$ mass splitting and to the energy difference between $\mathrm{d}$ and s-states. The latter is typically $330 \mathrm{MeV}$ which is too small with regard to the energies of lowlying nucleon and $\Delta$ excitations. Anyhow, the result represents only a small fraction of the pion contribution.

Our results for the ratios $E 2 / M 1$ and $C 2 / M 1$ are presented in Figs. 1 and 2. The E2 amplitude is calculated using (11). For the exact solutions of the model, both (11) and (10) should lead to the same result; using approximate methods to describe the nucleon and the $\Delta$, it turns out that expression (11) is much less sensitive to approximations than (10). This finding is well known, particularly in nuclear physics as the 'Siegert theorem'; in the context of elec- 


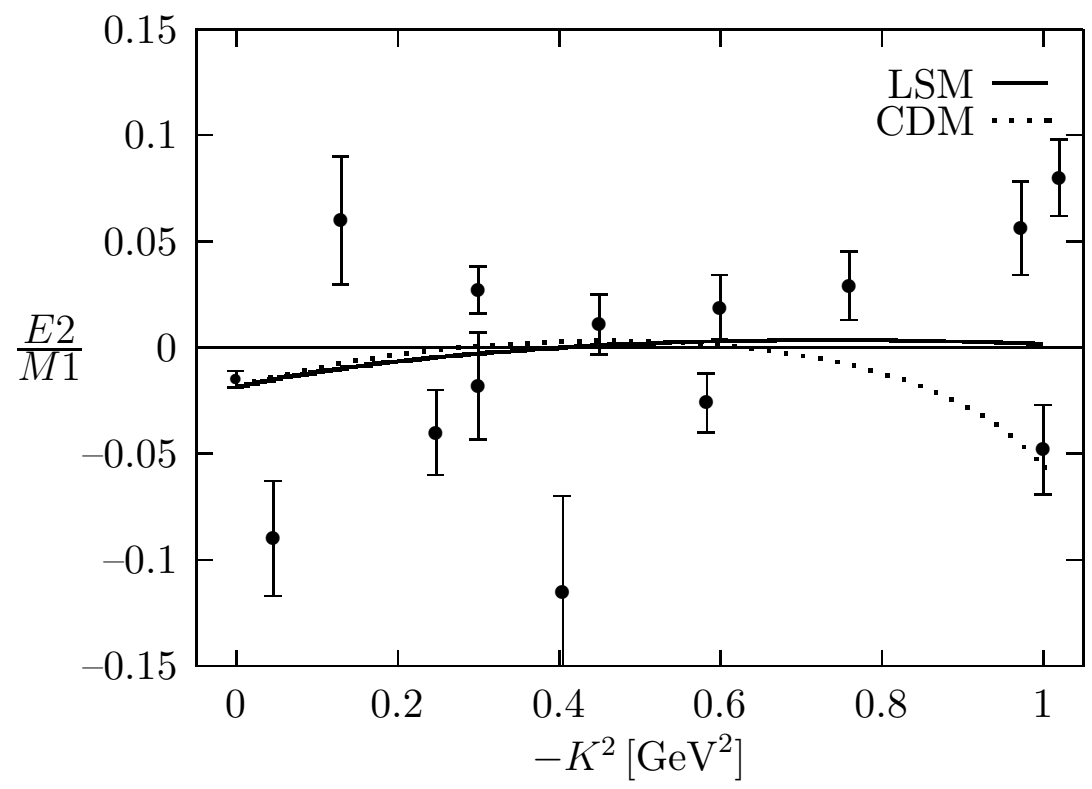

Fig. 1. The $E 2 / M 1$ ratio calculated in the linear $\sigma$-model for $g=5.0$ and $\varepsilon_{\Delta \mathrm{N}}=0.43 \mathrm{GeV}$ (full line), and in the chromodielectric model with quartic potential for $g=0.03 \mathrm{GeV}, M_{\chi}=1.33 \mathrm{GeV}$ and $\varepsilon_{\Delta \mathrm{N}}=0.33 \mathrm{GeV}$ (dotted line). Experimental points are taken from 13 15.

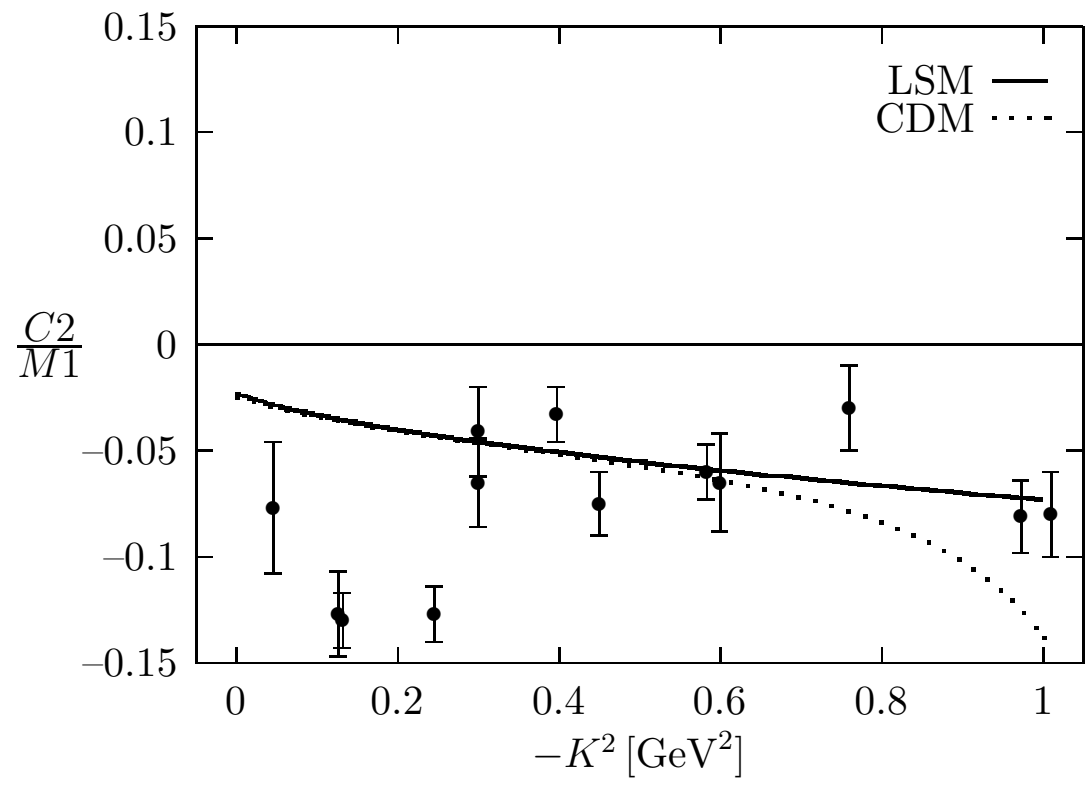

Fig. 2. The $C 2 / M 1$ ratio; for explanation see Fig. 1. Experimental points are taken from [14, 16].

troproduction of the $\Delta$, a similar conclusion has been discussed in Ref. 11]. The second term in (11) is very small and can be neglected; however, substituting $\frac{\partial}{\partial r} r j_{2}(k r)$ by $3 j_{2}(k r)$ as is usually done by assuming the small $k$ limit is not justified as a consequence of a large contribution from the pion tail. This is the main origin for different values of $E 2 / M 1$ and $C 2 / M 1$ at $K^{2}=0$ which are otherwise equal in the limit $k \rightarrow 0$. The computed value for $E 2 / M 1$ 
is $-1.9 \%$ in the CDM, and $-1.8 \%$ in the LSM, in good agreement with the experimental data $-1.5 \pm 0.4 \%$ 13 - though this value may be even higher in view of the recent measurements in Mainz [17] which give $-2.4 \pm 0.2 \%$. The ratios $C 2 / M 1$ are correspondingly $-2.5 \%$ and $-2.3 \%$ for the CDM and LSM. The behaviour of both ratios as a function of $K^{2}$ is consistent with still very uncertain experimental data. The quark contributions to the quadrupole amplitudes at the photon point are less than $10 \%$ with respect to the pion ones, and raise to around $15 \%$ at $K^{2}=-1 \mathrm{GeV}^{2}$ (for $C 2$ ); increasing ad hoc the d-s splitting to a more realistic value of $500 \mathrm{MeV}$ decreases these values by more than a factor of two.

The situation is not so favourable when considering the absolute values of the helicity amplitudes, $A_{1 / 2}$ and $A_{3 / 2}$ at $K^{2}=0$. In the LSM, the amplitudes (in units of $10^{-3} \mathrm{GeV}^{-1 / 2}$ ) are too small, -107 and -199 , respectively, compared to the experimental values $-141 \pm 7$ and $-259 \pm 10$ [13]. The contribution from pions is $50 \%$. In the CDM they are even smaller, -70 and -131 , respectively. In Fig. 3 we plot the behaviour of $M 1\left(K^{2}\right)=-\left[3 A_{3 / 2}+\sqrt{3} A_{1 / 2}\right] / 2 \sqrt{3}$, which is directly related to the magnetic $\Delta$ transition form factor $G_{\mathrm{M}}^{*}\left(-K^{2}\right)$ [2]. Except for the above discussed discrepancy for $K^{2} \rightarrow 0, M 1$ is well reproduced in the LSM, but not in the CDM where it rapidly decreases almost to zero at $K^{2}=-1 \mathrm{GeV}^{2}$. The latter is a consequence of the fact that the $M 1$, $E 2$ and $C 2$ transition densities are concentrated at the surface (a similar situation occurs also in the MIT model (for $M 1$ ) and in the CBM). At $K^{2}=$ $-1 \mathrm{GeV}^{2}$ the first zeros of $j_{1}(k r)$ and $j_{2}(k r)$ already come in the interior of the baryon and cancellation occurs, however in such a way that the ratios are almost unaffected. As our models suggest, the $C 2$ amplitude is the most clear manifestation of the presence of pions in the baryon; its large value at $K^{2}=-1 \mathrm{GeV}^{2}$ may therefore indicate a strong pion cloud in the interior. Such a strong cloud is predicted only in the LSM but not in the CDM. At $K^{2}=0$, however, the amplitudes $E 2$ and $C 2$ only 'see' the asymptotic pion field whose behaviour is determined by the Yukawa form and the $\pi \mathrm{NN}$ coupling constant, which is reasonably well reproduced in both models.

To the best of our knowledge this is the first calculation of the electroproduction quadrupole amplitudes in models with the pion cloud; other calculations all refer only to the photoproduction case. Apart from the already mentioned calculations in the CBM [4,5], the ratio E2/M1 was calculated in the Skyrme model [19] where almost a factor of two larger value was obtained. In Ref. [20], where the NJL model was used, the effect of the pion cloud does not appear explicitly, but rather through the excitations of the Dirac sea. The value for $E 2 / M 1$ they obtained is very close to our predictions (taking into account that they actually used the formula for $C 2 / M 1$ ). The constituent quark model has been used to describe both photoproduction and electroproduction. The predicted values for the ratios $E 2 / M 1$ and $C 2 / M 1$ are much smaller than the experimental ones - a clear indication that they cannot be reproduced in terms 


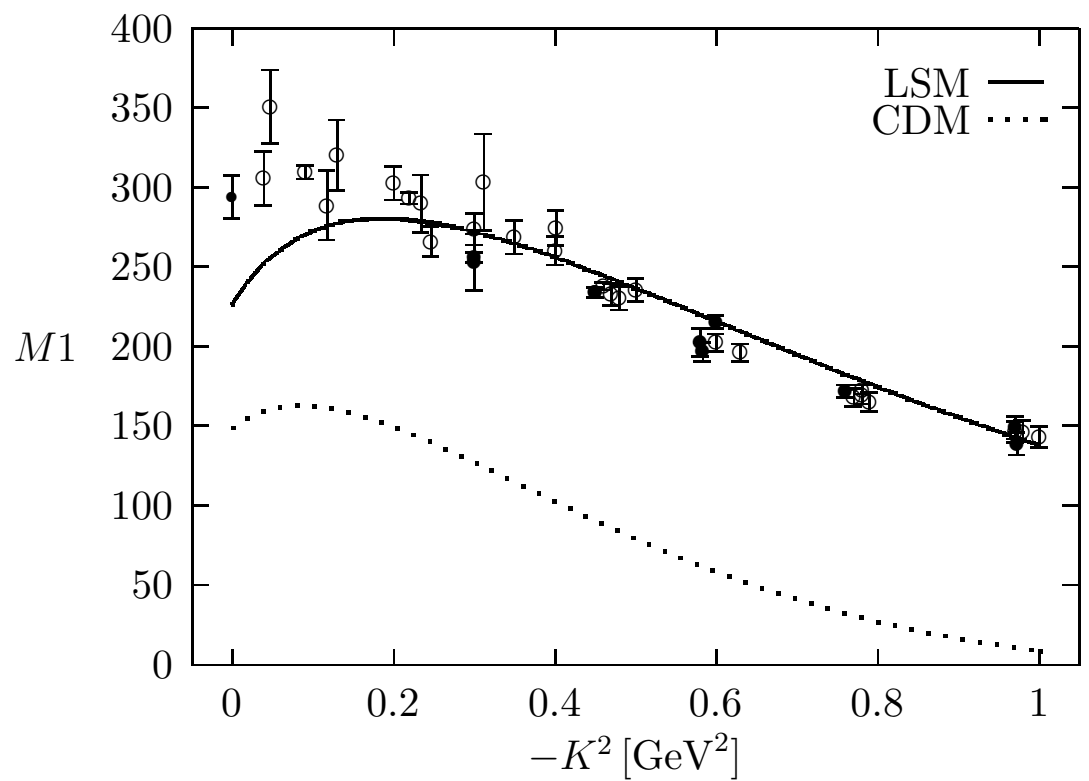

Fig. 3. The electromagnetic amplitude $M 1=-\left[3 A_{3 / 2}+\sqrt{3} A_{1 / 2}\right] / 2 \sqrt{3}$ in units of $10^{-3} \mathrm{GeV}^{-1 / 2}$ calculated in the linear $\sigma$-model and chromodielectric model (see Fig. 1). The experimental value at the photon point is taken from [13. For virtual photons, the values denoted by $\bullet$ are determined from the $\left|M_{1+}\right|$ amplitude for the process $\gamma \mathrm{p} \rightarrow \mathrm{p} \pi^{0}$ [14] assuming the dominance of the $T=3 / 2$ amplitude, the values denoted by $\circ$ are computed from $G_{\mathrm{M}}^{*}$ [15, 18].

of valence quarks alone. It is interesting to notice that a good estimate for the $E 2 / M 1$ ratio can be obtained in the old CGLN model [21] in which the nucleon and the $\Delta$ are described as a bare nucleon surrounded by a pion cloud having a Yukawa form with a cut-off in momentum space. Using their formulas for $E_{1+}$ and $M_{1+}$ and neglecting the crossed term one obtains a value around $-2 \%$, almost insensitive to the cut-off momentum. This strongly supports our conclusion that in photoproduction the ratio is essentially determined by the tail of the pion cloud.

It is instructive to analyze the results for the production amplitudes in terms of the relation $\mu_{\Delta \mathrm{N}}=\frac{2}{3} \mu_{\mathrm{v}} g_{\pi \Delta \mathrm{N}} / g_{\pi \mathrm{NN}}$, where the $g$ 's are the $\pi \Delta \mathrm{N}$ and $\pi \mathrm{NN}$ coupling constants, $\mu_{\mathrm{v}}$ is the isovector part of the nucleon magnetic moment, and $\mu_{\Delta \mathrm{N}}$ is the transition magnetic moment which is proportional to the M1 amplitude (9) in the limit $k \rightarrow 0$. This relation is trivially fulfilled in quark models, but, as shown already in Ref. [21], it is quite general and model independent. In models with only valence quarks, the ratio of the coupling constants is $\sqrt{72 / 25}$ which is some $25 \%$ below the value deduced from the experiment. It is therefore not surprising that almost all models where the valence quarks play a dominant role, predict too low values for the photoproduction amplitudes, even if they otherwise reproduce the nucleon magnetic moments. Furthermore, in relativistic quark models with no pions or with a weak pion field, such as the CDM, the nucleon magnetic moments are generally underestimated; both effects may explain small values for photoproduction amplitudes obtained in 
such models. In the MIT model [22] a bag radius as big as $1.4 \mathrm{fm}$ was used to obtain the value $-102 \cdot 10^{-3} \mathrm{GeV}^{-1 / 2}$ for $A_{1 / 2}$; decreasing the radius to a more realistic $1 \mathrm{fm}$ would bring $A_{1 / 2}$ down to only $1 / 2$ of the experimental value, in agreement with our result for the CDM. Possible improvements, such as the linear momentum projection, increase the magnetic moments [23] and very likely also the production amplitudes, though probably not to the extent that they would cure considerably the disagreement in this type of models. In the LSM, the nonlinear effects from the Mexican hat potential increase the ratio of the coupling constants to 2.05 which agrees well with the experimentally deduced value. Since $\mu_{\mathrm{v}}$ is also close to the experimental value, this may explain good overall agreement in Fig. 3 except for the mentioned discrepancy at very low $K^{2}$. This discrepancy still remains an open question; let us just mention that it is inherent not only to this model since a very similar behaviour was found in a recent calculation in the constituent quark model using the light-front approach [3].

In summary, we conclude that the pion cloud plays an important role in the electroproduction of the $\Delta$ resonance. It yields the major contribution to the quadrupole amplitudes which show a consistent behaviour also for $K^{2} \neq 0$. Furthermore, it may considerably contribute to the absolute values of the production amplitudes and may, as in the LSM, yield a good agreement with the experimental results for $-K^{2}>0.2 \mathrm{GeV}^{2}$. Yet, it still does not resolve the problem why almost all theoretical predictions underestimate the experimental values at $K^{2}=0$. We have also shown that the results at the photon point are rather insensitive to the details of the model; in order to be able to test different models it is necessary to go to nonzero $K^{2}$. Here, large values of the $C 2$ amplitude may be an important indication of the presence of a strong pion cloud in the interior of the nucleon and the $\Delta$.

BG and SŠ would like to acknowledge the hospitality they enjoyed during a visit to the Department of Physics of the University of Coimbra and MF the hospitality at J. Stefan Institute. This work was supported by the Calouste Gulbenkian Foundation (Lisbon), the Ministry of Science of Slovenia, and the European Commission contract ERB-CIPA-CT-92-2287.

\section{References}

[1] S. Capstick and G. Karl, Phys. Rev. D 41 (1990) 2767; S. Capstick, Phys. Rev. D 46 (1992) 2864; F. E. Close and Zhenping Li, Phys. Rev. D 42 (1990) 2194, 2207; E. Eich, Z. Phys. C 39 (1988) 399; H. J. Weber, Ann. Phys. (N.Y.) 207 (1991) 417.

[2] M. Warns et al., Z. Phys. C 45 (1990) 627; Phys. Rev. D 42 (1990) 2215. 
[3] S. Capstick and B. D. Keister, Phys. Rev. D 51 (1995) 3598.

[4] K. Bermuth et al., Phys. Rev. D 37 (1988) 89.

[5] G. Kälbermann and J. M. Eisenberg, Phys. Rev. D 28 (1983) 71.

[6] M. C. Birse, Progr. Part. Nucl. Phys. 25 (1990) 1.

[7] A. Drago, M. Fiolhais and V. Tambini, Nucl. Phys. A 588 (1995) 801.

[8] M. C. Birse and M. K. Banerjee, Phys. Lett. B 136 (1984) 284; Phys. Rev. D 31 (1985) 118; S. Kahana, G. Ripka and V. Soni, Nucl. Phys. A 415 (1984) 351.

[9] B. Golli and M. Rosina, Phys. Lett. B 165 (1985) 347; M. C. Birse, Phys. Rev. D 33 (1986) 1934.

[10] M. Kim and M. K. Banerjee, Phys. Rev. C 48 (1993) 2035.

[11] M. Bourdeau and N. C. Mukhopadhyay, Phys. Rev. Lett. 58 (1987) 976.

[12] M. Čibej, M. Fiolhais, B. Golli and M. Rosina, J. Phys. G. 18 (1992) 49.

[13] Particle Data Group, Phys. Rev. D 50 (1994) 1712.

[14] R. Siddle et al., Nucl. Phys. B 35 (1971) 93; J. C. Alder et al., Nucl. Phys. B 46 (1972) 573; K. Bätzner et al., Nucl. Phys. B 76 (1974) 1.

[15] S. Galster et al., Phys. Rev. D 5 (1972) 519; C. Mistretta et al., Phys. Rev. 184 (1969) 1487.

[16] R. L. Crawford, Nucl. Phys. B 28 (1971) 573; F. Kalleicher, PhD Thesis KPH 8/94, Universität Mainz, Mainz 1993.

[17] R. Beck, Talk presented at the Baryons '95 Conference, Santa Fe, 1995.

[18] W. W. Ash et al., Phys. Lett. B 24 (1967) 165; S. Stein et al., Phys. Rev. D 12 (1975) 1884; S. Galster et al., Phys. Rev. D 5 (1972) 519; W. Bartel et al., Phys. Lett. B 28 (1968) 148.

[19] A. Wirzba and W. Weise, Phys. Lett. B 188 (1987) 6.

[20] T. Watabe, Chr. V. Christov, K. Goeke, Phys. Lett. B 349 (1995) 197.

[21] G. F. Chew et al., Phys. Rev. 106 (1957) 1345.

[22] J. F. Donoghue et al., Phys. Rev. D 12 (1975) 2875.

[23] T. Neuber et al., Nucl. Phys. A 560 (1993) 906. 